\title{
Mapping interactions among cell-free expressed Zika virus proteins
}

\author{
Shayli Varasteh Moradi ${ }^{1}$, Dejan Gagoski ${ }^{2}$, Sergey Mureev ${ }^{1}$, Patricia Walden ${ }^{1}$, Kerrie-Ann \\ McMahon $^{3}$, Robert G. Parton ${ }^{3,4}$, Wayne A Johnston ${ }^{1}$ and Kirill Alexandrov ${ }^{1, *}$ \\ ${ }^{1}$ CSIRO-QUT Synthetic Biology Alliance, Centre for Tropical Crops and Biocommodities, Queensland \\ University of Technology, Brisbane, QLD 4001, Australia \\ ${ }^{2}$ Queensland Brain Institute, The University of Queensland, St Lucia, QLD 4072, Queensland, Australia \\ ${ }^{3}$ Institute for Molecular Bioscience, The University of Queensland, St Lucia, QLD 4072, Australia \\ ${ }^{4}$ Centre for Microscopy and Microanalysis, The University of Queensland, St Lucia, QLD 4072, \\ Queensland, Australia
}

\section{Supporting Information}

\section{A) Gel images and schematic figure of AlphaLISa assay design}

Fig. S1. SDS-PAGE analysis of the LTE expressed ZIKV proteins

Fig. S2. Bead pair design for detection of viral proteins tagged with fluorescent proteins in AlphaLISA assay

Fig. S3. Pull down analysis of ZIKV protein interactions

Fig. S4. Effect of salt concentration on Capsid protein interaction

Fig. S5. Comparison of AlphaLISA and Alphascreen signal

Table S1: Summary of viral protein-protein interaction in Flavivirus genus reported in literature

B) List of gene sequences used in this study (excel sheet file) 

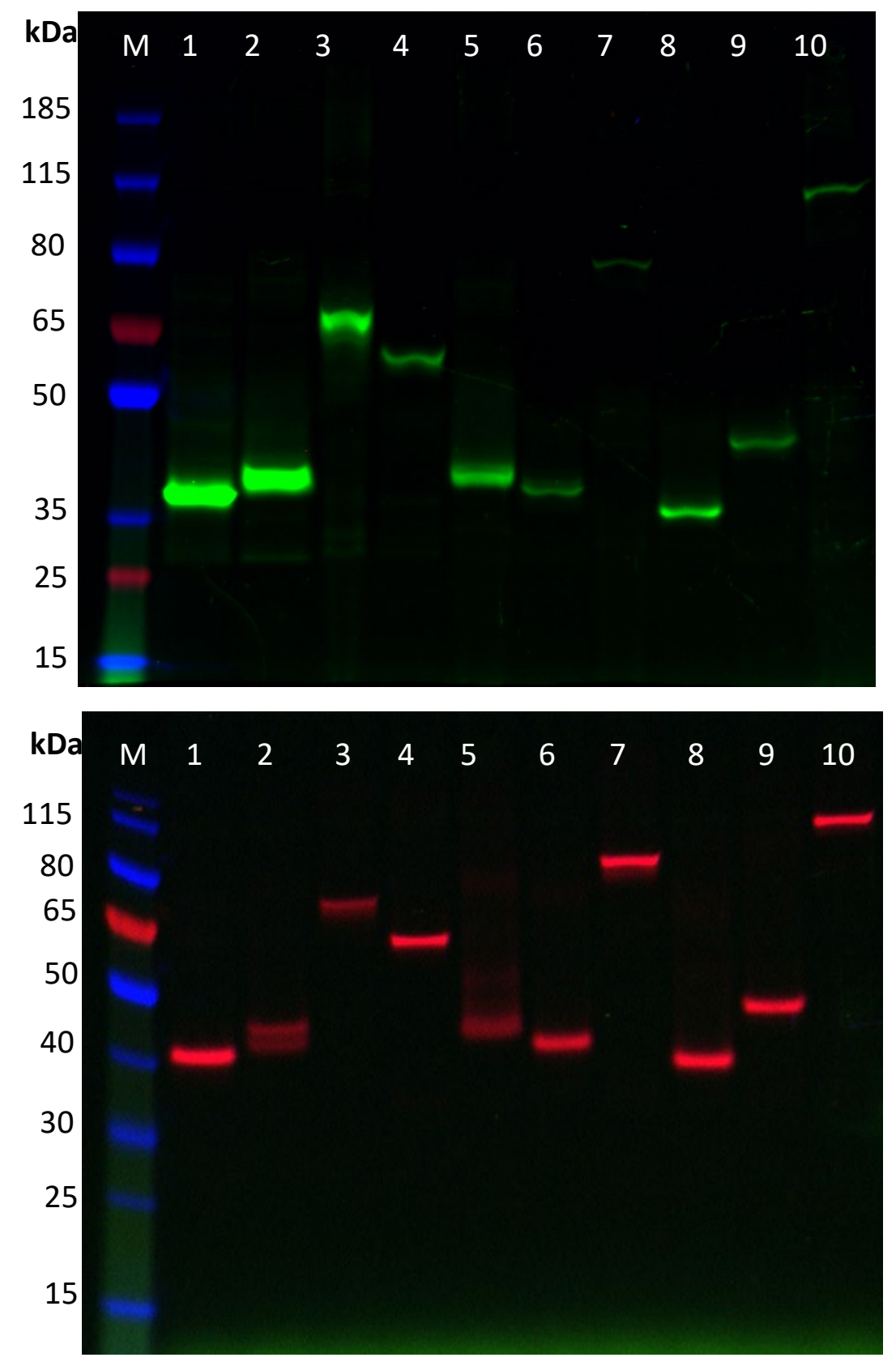

Fig. S1. SDS-PAGE analysis of the LTE expressed ZIKV proteins: The proteins were expressed as GFP and mCherry and separated on SDS-PAGE gel (4-12\% Tris-glycine) and visualized by in gel fluorescence scanning. M: marker; 1 Capsid; 2 PrM; 3 Envelope; 4 NS1; 5 NS2A; 6 NS2B; 7 NS3; 8 NS4A; 9 NS4B; 10 NS5 


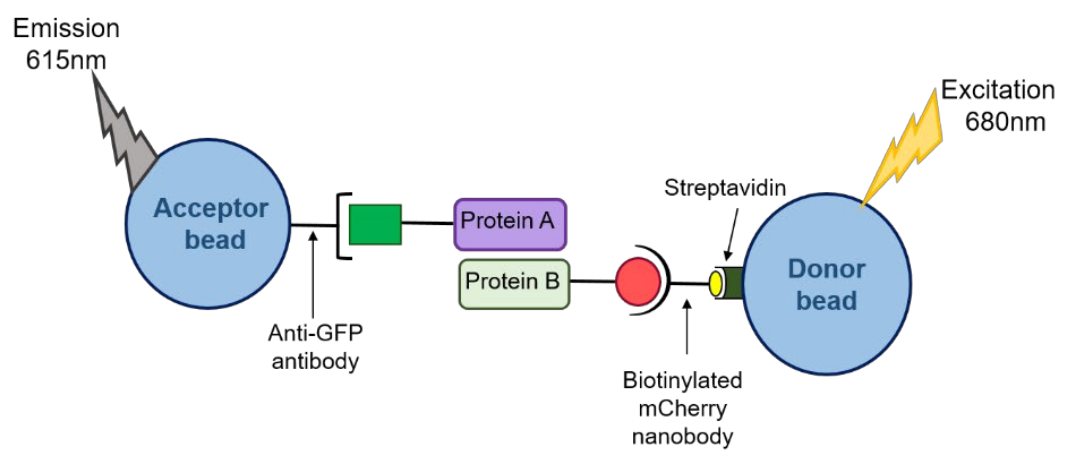

Fig. S2. Bead pair design for detection of viral proteins tagged with fluorescent proteins in AlphaLISA assay: The interaction between proteins was monitored by AlphaLISA in which the interacting proteins are captured with streptavidin coated donor beads coupled to anti-mCherry nanobody and anti-GFP antibody acceptor beads. The donor beads produce singlet oxygen upon excitation at $680 \mathrm{~nm}$ which is able to transfer energy to the acceptor beads; If the acceptor bead comes to the proximity of donor bead (maximum $200 \mathrm{~nm}$ ), the singlet oxygen reacts with thioxine derivative in the acceptor bead, subsequently emits luminescent light at $615 \mathrm{~nm}$. 
(A)

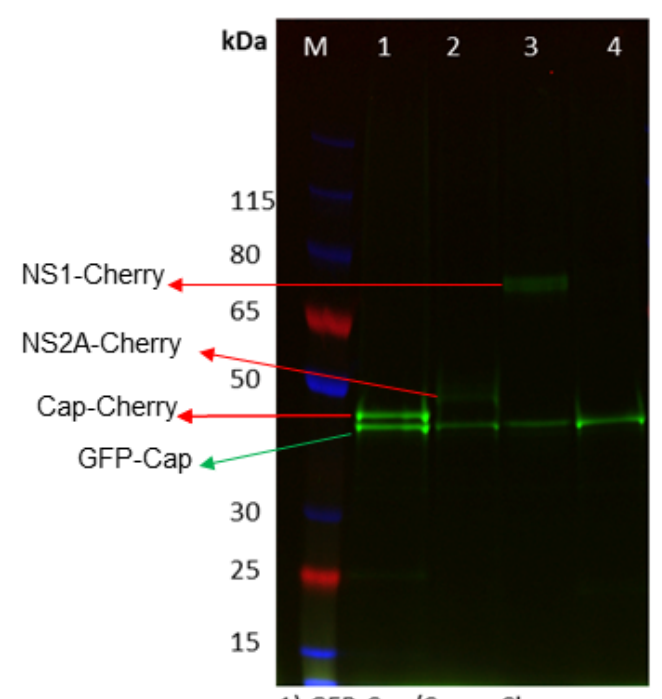

1) GFP-Cap/Cap-mCherry

2) GFP-Cap/NS2A-mCherry

3) GFP-Cap/NS1-mcherry

4) GFP-Cap/ FRB-mCherry

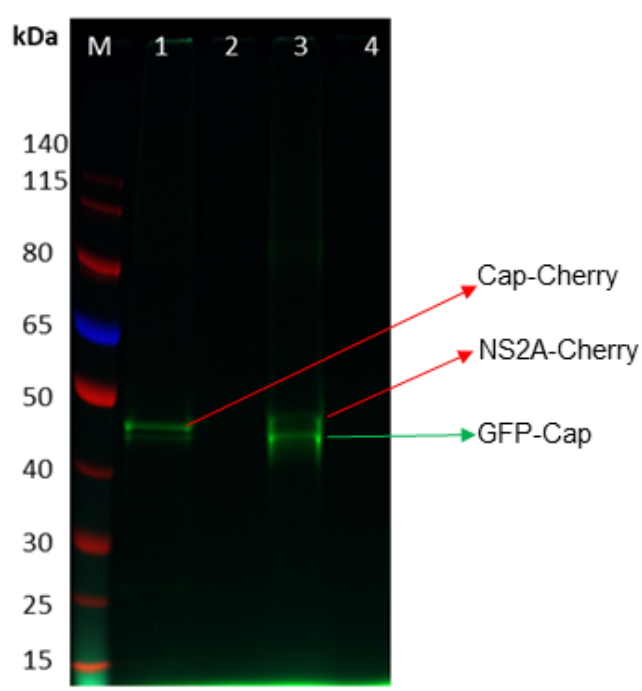

1) GFP-Cap/Cap-

mCherry

2) Cap-mCherry

3) GFP-Cap/NS2A-

mCherry

4) NS2A-mcherry
(B)

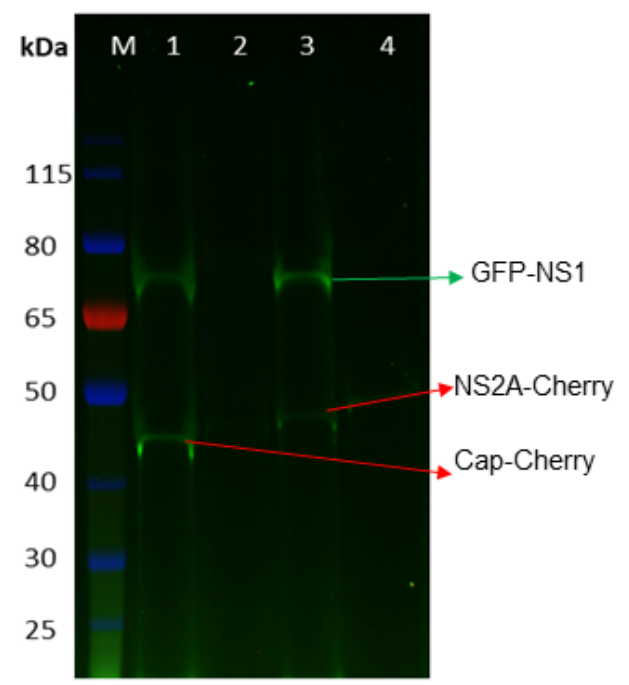

1) GFP-NS1/Cap-mCherry

2) Cap-mCherry

3) GFP-NS1/NS2A-mCherry

4) NS2A-mCherry
(C)

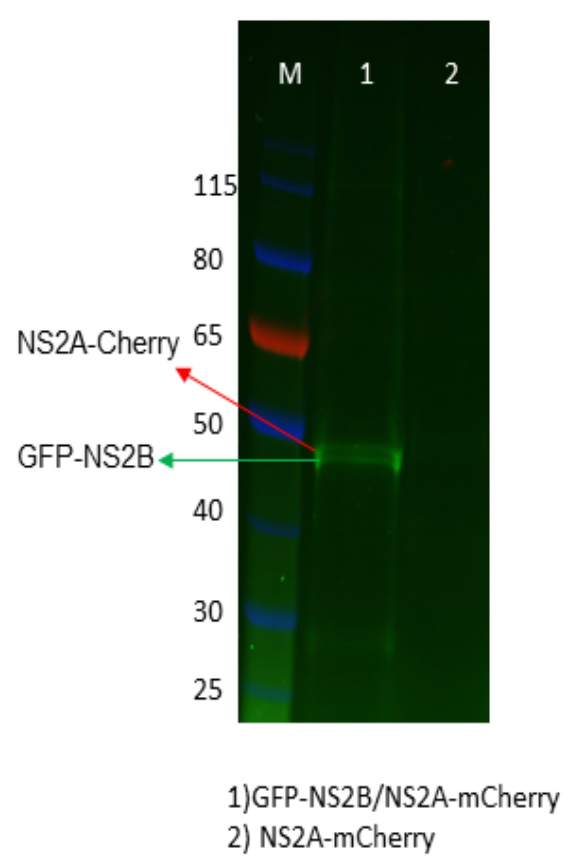

(D)

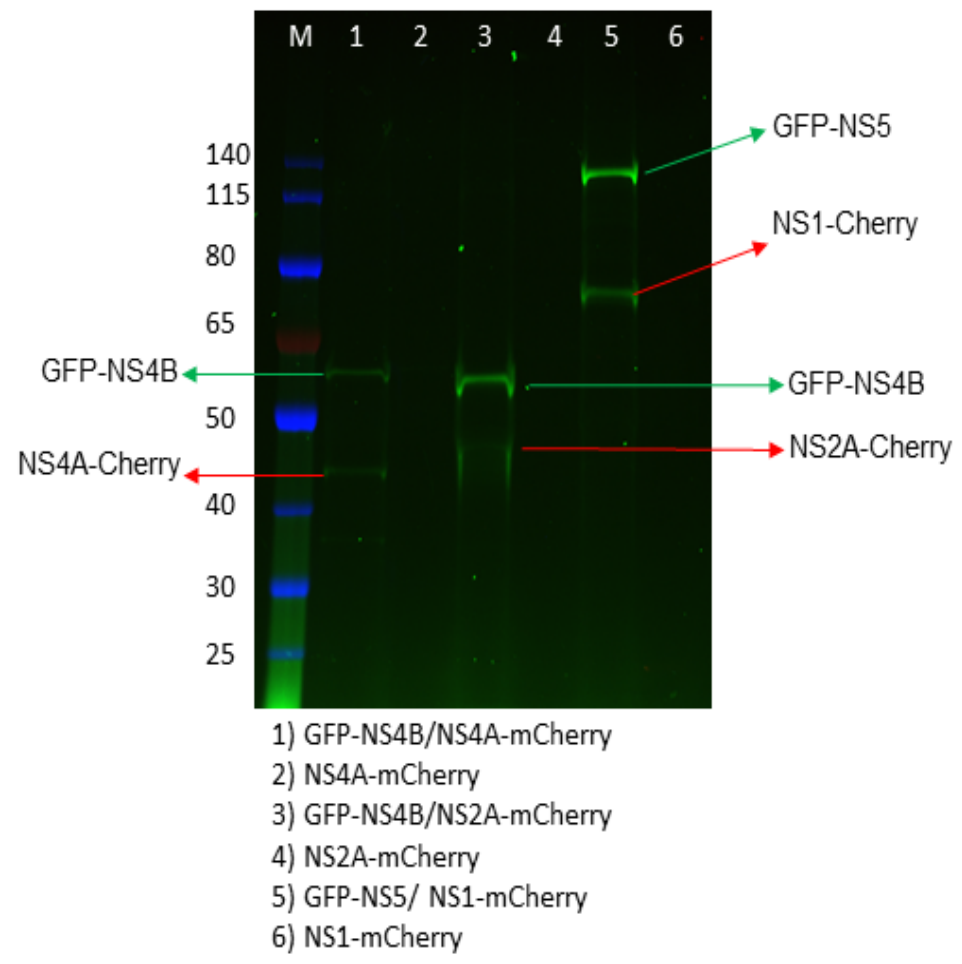


Fig. S3. Pull down analysis of ZIKV protein interactions. The protein pairs were co- expressed in LTE reaction containing 1:500 (V/V) dilution of BODIPY-Lys-tRNA and pulled down using GFP trap beads. The eluted protein pairs were resolved on SDS-PAGE gel and visualized by scanning the gel without staining. (A, B, C and D) SDS-PAGE images of ZIKV protein pairs isolated by GFP trap beads. The protein pairs in each lane are labeled under each image. FRB protein in image (A) was used as non interacting partner of Cap protein (negative control).

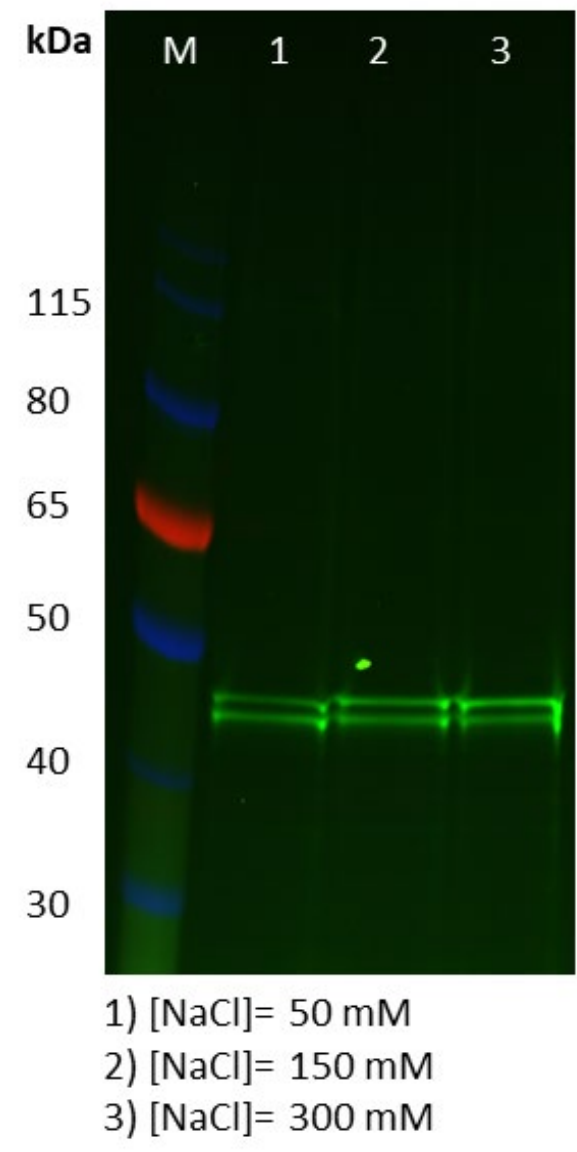

Fig. S4. Effect of salt concentration on Capsid protein interaction. GFP nanotrap beads were added to the protein expressing reactions concurrently. Following completion of reaction, the 
bound proteins were washed four times with different washing buffers containing 50, 150, 300 $\mathrm{mM}$ of NacL. The interaction of capsid proteins was not affected with changing salt concentration

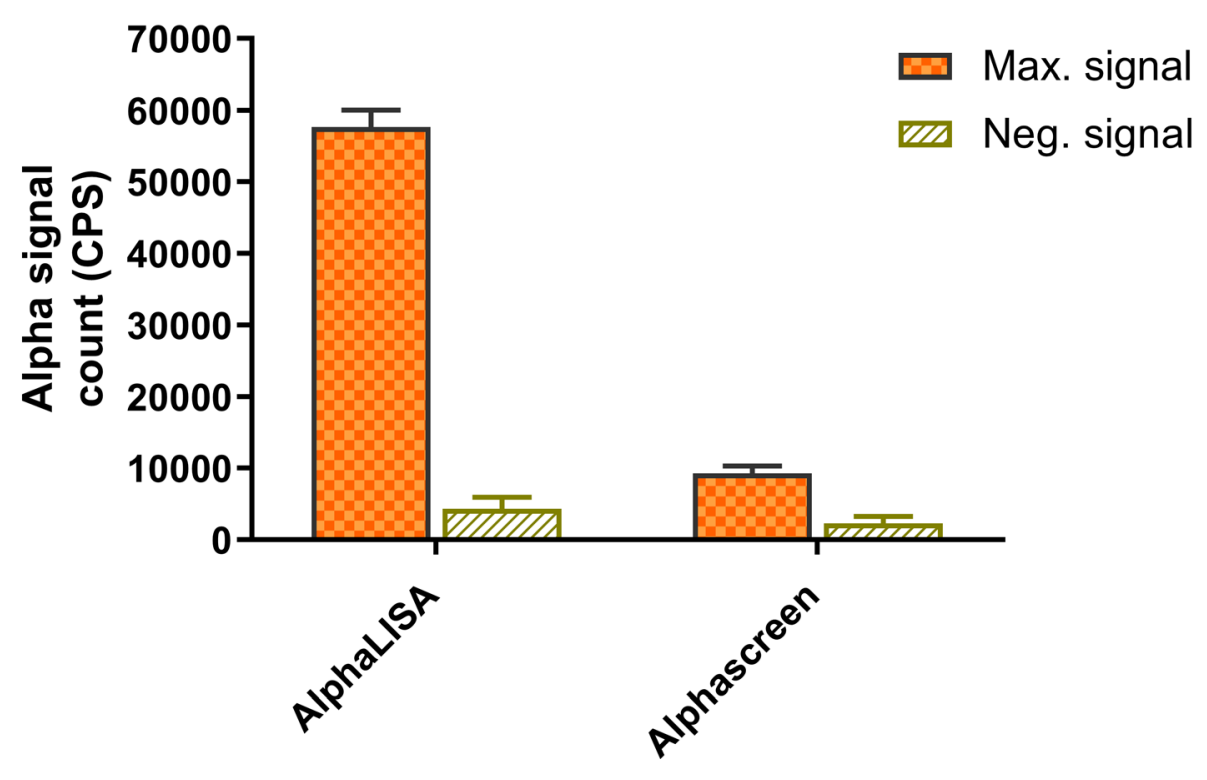

Fig. S5. Comparison of AlphaLISA and Alphascreen signal: Capsid protein tagged with GFP and mCherry was used in the comparison study. The negative control experiment was performed with GFP- FRB and Capsid-mCherry proteins. All assay conditions were maintained constant with both AlphaLISA and Alphascreen beads. The alpha signal obtained by AlphaLISA and AlphaScreen is 12.2 and 4 times higher than background signal respectively. The assay showed that the AlphaLISA beads produce more reliable signals. 
Table 1: Summary of viral protein-protein interaction in Flavivirus genus reported in literature

\begin{tabular}{|c|l|l|c|}
\hline Virus & Interacting proteins & \multicolumn{1}{|c|}{ Interaction detection method } & Reference \\
\hline $\begin{array}{c}\text { West Nile } \\
\text { virus }\end{array}$ & $\begin{array}{l}\text { NS2A-NS4A; } \\
\text { NS2B-NS2A; NSB- } \\
\text { NS4A; NS2B-NS3; } \\
\text { NS3-NS5, } \\
\text { NS1dimer, NS3 } \\
\text { dimer }\end{array}$ & $\begin{array}{l}\text { Imaging-based techniques: fluorescence } \\
\text { resonanceenergytransfer (FRET), } \\
\text { biologic } \\
\text { fluorescencecomplementation(BiFC). }\end{array}$ & $\mathbf{1}$ \\
\hline $\begin{array}{c}\text { Dengue } \\
\text { virus type 2 } \\
\text { (DENV-2) }\end{array}$ & $\begin{array}{l}\text { NS4A dimer and } \\
\text { oligomer }\end{array}$ & Coimmunoprecipitation (Co-IP) & $\mathbf{2}$ \\
\hline $\begin{array}{c}\text { Dengue } \\
\text { virus }\end{array}$ & NS1-Envelope & $\begin{array}{l}\text { Western-blot and immunoprecipitation } \\
\text { analysis }\end{array}$ & $\mathbf{3}$ \\
\hline DENV-2 & NS3-NS4B & Proximity ligation assay, Co-IP & $\mathbf{4}$ \\
\hline Zika virus & NS2B-NS3 & Crystal structure & $\mathbf{6}$ \\
\hline $\begin{array}{c}\text { Dengue } \\
\text { virus }\end{array}$ & NS2A-NS2B & $\begin{array}{l}\text { Intermolecular interactions at genetic } \\
\text { level using immunofluorescence analysis } \\
\text { (IFA) and plaque-forming assays }\end{array}$ & $\mathbf{7}$ \\
\hline $\begin{array}{c}\text { DENV-2 } \\
\text { NS2A dimer, }\end{array}$ & Western blot & $\mathbf{8}$ \\
\hline Zika virus & Capsid dimer & Crystal structure & $\mathbf{9}$ \\
\hline Zika virus & PrM-Envelope & Crystal structure & $\mathbf{1 0}$ \\
\hline Zika virus & NS1 dimer & Crystal structure & $\mathbf{1 1}$ \\
\hline DENV-2 & NS4B dimer & Proximity ligation assay, Co-IP & cross-linking and pull-down \\
\hline DENV-2 & NS4A-NS4B & Nentamer & \\
\hline
\end{tabular}




\section{References:}

1. Yu, L.; Takeda, K.; Markoff, L., Protein-protein interactions among West Nile non-structural proteins and transmembrane complex formation in mammalian cells. Virology 2013, 446 (1), 365-377.

2. Lee, C. M.; Xie, X.; Zou, J.; Li, S. H.; Lee, M. Y.; Dong, H.; Qin, C. F.; Kang, C.; Shi, P. Y., Determinants of Dengue Virus NS4A Protein Oligomerization. J Virol 2015, 89 (12), 6171-83.

3. Scaturro, P.; Cortese, M.; Chatel-Chaix, L.; Fischl, W.; Bartenschlager, R., Dengue virus nonstructural protein 1 modulates infectious particle production via interaction with the structural proteins. PLoS pathogens 2015, 11 (11), e1005277.

4. Zou, J.; Wang, Q. Y.; Xie, X.; Lu, S.; Yau, Y. H.; Yuan, Z.; Shochat, S. G.; Kang, C.; Lescar, J.; Shi, P.-Y., Mapping the interactions between the NS4B and NS3 proteins of dengue virus. Journal of virology 2015, 89 (7), 3471-3483.

5. Lei, J.; Hansen, G.; Nitsche, C.; Klein, C. D.; Zhang, L.; Hilgenfeld, R., Crystal structure of Zika virus NS2B-NS3 protease in complex with a boronate inhibitor. Science 2016, 353 (6298), 503.

6. Wu, R.-H.; Tsai, M.-H.; Tsai, K.-N.; Tian, J. N.; Wu, J.-S.; Wu, S.-Y.; Chern, J.-H.; Chen, C.-H.; Yueh, A. J. J. o. v., Mutagenesis of dengue virus protein NS2A revealed a novel domain responsible for virus-induced cytopathic effect and interactions between NS2A and NS2B transmembrane segments. 2017, 91 (12), e01836-16.

7. Shrivastava, G.; García-Cordero, J.; León-Juárez, M.; Oza, G.; Tapia-Ramírez, J.; VillegasSepulveda, N.; Cedillo-Barrón, L., NS2A comprises a putative viroporin of Dengue virus 2. Virulence 2017, 8 (7), 1450-1456.

8. $\quad$ Li, T.; Zhao, Q.; Yang, X.; Chen, C.; Yang, K.; Wu, C.; Zhang, T.; Duan, Y.; Xue, X.; Mi, K.; Ji, X.; Wang, Z.; Yang, H., Structural insight into the Zika virus capsid encapsulating the viral genome. Cell Research 2018, 28 (4), 497-499.

9. Prasad, V. M.; Miller, A. S.; Klose, T.; Sirohi, D.; Buda, G.; Jiang, W.; Kuhn, R. J.; Rossmann, M. G., Structure of the immature Zika virus at $9 \AA$ resolution. Nature Structural and Molecular Biology 2017, 24 (2), 184.

10. Brown, W. C.; Akey, D. L.; Konwerski, J. R.; Tarrasch, J. T.; Skiniotis, G.; Kuhn, R. J.; Smith, J. L., Extended surface for membrane association in Zika virus NS1 structure. Nature Structural \& Molecular Biology 2016, 23 (9), 865-867.

11. Zou, J.; Xie, X.; Chandrasekaran, R.; Reynaud, A.; Yap, L.; Wang, Q.-Y.; Dong, H.; Kang, C.; Yuan, Z.; Lescar, J., Dimerization of flavivirus NS4B protein. Journal of virology 2014, JVI. 02782-13.

12. Zou, J.; Xie, X.; Wang, Q.-Y.; Dong, H.; Lee, M. Y.; Kang, C.; Yuan, Z.; Shi, P.-Y., Characterization of dengue virus NS4A and NS4B protein interaction. Journal of virology 2015, 89 (7), 3455-3470. 